\title{
1
}

\section{Commercial and Public Use Applications}

\author{
Dr. Hariharan Krishnan and Dr. Fan Bai
}

General Motors

\section{Dr. Gavin Holland}

\author{
HRL Laboratories, LLC
}

Together, the Dedicated Short Range Communications (DSRC) and Vehicular Ad hoc Network (VANET) technologies provide a unique opportunity to develop various types of communication-based automotive applications. In this chapter, we focus primarily on four major aspects: (a) description of communication-based automotive applications, (b) investigating the application characteristics and network attributes, (c) classifying the applications into categories, and (d) defining market perspectives and deployment challenges for each class of applications. To date, many applications have been identified by the automotive research community. From a value or customer benefit perspective, these applications can be roughly organized into three major classes: safety-oriented, convenience-oriented, and commercial-oriented, and they vary significantly in terms of application characteristics. We begin by describing communication-based automotive applications that span both the Vehicle-to-Vehicle (V2V) and Vehicle-to-Infrastructure (V2I) communication modes. We follow a systematic classification methodology for such applications that goes through two major steps: characterization and classification. We focus on a rich subset of representative applications and characterize them with respect to plausible application- and networkingrelated attributes. The characterization process not only strengthens our understanding of the applications but also sets the stage for the classification step, since it reveals numerous

Vehicular Networking Edited by Marc Emmelmann, Bernd Bochow, C. Christopher Kellum

(C) 2010 John Wiley \& Sons, Ltd 
application commonalities. The applications are classified into several generic classes, with the consideration of balancing the trade-off between exploiting as many application similarities as possible and preserving their salient differences. Such classification is of paramount importance in bridging the gap between the automotive and wireless networking communities. We also try and define market perspectives and deployment challenges for each representative class of applications.

\subsection{Introduction}

The needs for significant reduction in both highway traffic congestion and vehicle crashes are serious challenges throughout the world (Chen and Cai 2005; Reumerman et al. 2005). In order to address these challenges, expensive sensors, radars, cameras, and other stateof-the-art technologies are currently integrated into vehicles to improve vehicle safety and driver comfort during travel. Recently, communication-based applications based on Vehicleto-Vehicle/Infrastructure (V2X) communications have attracted more attention from industry and governments in the United States, Europe, Japan, and Australia, because of their unique potential to address vehicle safety and traffic congestion challenges at lower operational costs (Sengupta et al. 2007; VSCC 2006). In addition to safety and traffic efficiency applications, wireless communication can also be shared by commercial and vehicular 'infotainment' applications to, for instance, enhance the occupants' driving experience. Thus, wireless communication can be used not only to enhance transportation safety (ElBatt et al. 2006; Torrent-Moreno et al. 2004; Xu et al. 2004; Yin et al. 2004) and traffic efficiency (Anda et al. 2005), but also to create commercial value to vehicle owners and automotive Original Equipment Manufacturers (OEMs) by providing infotainment applications (Das et al. 2004; Nandan et al. 2005).

The United States Department of Transportation (DOT) has recognized the importance of having a dedicated wireless spectrum for improving vehicle safety and traffic efficiency. Hence, in the USA, the Federal Communications Commission (FCC) has allocated $75 \mathrm{MHz}$ of licensed spectrum at $5.9 \mathrm{GHz}$ as the DSRC band for Intelligent Transportation System (ITS) services (FCC 2003). This is used in the rest of North America also. In Europe, the Commission of the European Communities has specified harmonized use of radio spectrum in the 5875-5905 MHz frequency band for safety related applications of ITS. In Japan, the deployment of Electronic Toll Collection (ETC) uses the $5.8 \mathrm{GHz}$ spectrum. The future plan is to expand the $5.8 \mathrm{GHz}$ spectrum use for V2I communication-based applications, and the $700 \mathrm{MHz}$ spectrum for $\mathrm{V} 2 \mathrm{~V}$ communication-based applications. The Australian government is considering a similar allocation of radio spectrum at $5.9 \mathrm{GHz}$ for ITS. The deployment of ITS, with its V2V and V2I constituents, is supported under major DOT initiatives (CICAS 2009; VII 2009). The Physical (PHY) and Medium Access Control (MAC) portions of the DSRC standard are currently being addressed by the IEEE $802.11 \mathrm{p}$ Task Group (IEEE 802.11 1999; IEEE P802.11p/D6.0 2009), which is widely considered as the leading technology for communication-based automotive applications. Major automotive OEMs, wireless device manufacturers, research institutions, public agencies, and private enterprises are conducting research on various topics pertaining to V2X communications, such as wireless channel modeling (Taliwal et al. 2004; Yin et al. 2006), mobility modeling (Bai et al. 2003; Lin et al. 2004), routing protocols (Chennikara-Varghese et al. 2006; Korkmaz et al. 2004; LeBrun et al. 2005; Lochert et al. 2005), security (Picconi et al. 2006; 
Raya et al. 2006), and market penetration mitigation strategies (Kosch 2005; Shladover and Tan 2006). There has also been some limited attention dedicated to better understanding, modeling and analyzing communication-based automotive applications as the major driving force for VANET-focused technologies.

\subsubsection{Motivation}

This work is motivated by the need for a systematic and thorough analysis of communicationbased automotive applications from a networking point of view. As a preliminary study, we attempt not only to raise awareness about the performance requirements of the automotive community, but also to attract sufficient attention from the networking research community.

The Vehicle Safety Communication Project (VSCC 2006) has identified a number of applications for potential deployment along with projected user benefits (VSCC 2005). The applications of interest vary significantly in terms of their characteristics, requirements, and constraints, ranging from safety/warning applications to content download/streaming applications (for entertainment) and free-flow payment applications (for improving highway traffic efficiency and driver convenience). Analyzing and developing wireless networking solutions tailored to such a large number of diverse applications, in an exhaustive manner, is a cumbersome and inefficient task. Obviously there is a gap between developing communication-based automotive applications (the focus of the automotive community) and developing VANET protocols (the focus of the wireless networking community). To bridge this void, we aim at categorizing communication-based automotive applications, not only from the application characteristics perspective, but more importantly from a wireless networking perspective.

\subsubsection{Contributions and benefits}

To the best of our knowledge, this chapter is the first study of classifying communicationbased automotive applications from the perspective of wireless networking design. Towards this objective, we are interested in answering the following questions:

(a) What are the key application characteristics and networking attributes in the design space of automotive application development?

(b) How should these applications be categorized into generic classes, from the viewpoint of network designers?

(c) What are the market perspectives and deployment challenges associated with each class of applications?

Part of the challenge in this study is to create a rich set of application characteristics and network attributes, which capture the major dimensions of the design space of V2X applications in a systematic and thorough manner. With deep insight into the application design space, we have categorized a set of applications into several generic classes based on their identified commonalities. We focus primarily on three major aspects: (a) investigating the application characteristics and network attributes, (b) classifying the applications into generic classes, and (c) understanding market perspectives and deployment challenges for each class of applications. 
Our aim for this study is not only to simplify large-scale simulation efforts, which play an important role in understanding the performance limits of VANETs in realistic scenarios, but also to shed light on designing network protocol stack(s) and system integration for different applications. For instance, using the analysis, network designers may focus on just a few abstract classes of V2X applications, rather than designing for individual applications in an exhaustive manner. Also, evaluating the performance trends of generic classes of applications with the same mechanisms or tools simplifies the task and reveals valuable insights at a reasonable cost. If necessary, individual applications can be further studied and analyzed as simple extensions of the proposed generic classes. Finally, it should be noted that the proposed classes are not meant to be comprehensive, but they constitute an essential first step that could be refined and extended in the future as automotive applications emerge, dominate or subside.

This classification serves as a potential road-map for developing the VANET technology needed to support different applications. A generic class of applications is more likely to have a similar set of protocols and mechanisms in the network stack because similar application characteristics and performance requirements tend to implicitly mandate the same technical solution. Thus, network designers should be able to maximize the reusability of common mechanistic 'building blocks' (or modules) for a specific class of applications with similar application characteristics and performance requirements.

\subsubsection{Chapter organization}

This chapter is organized as follows. In Section 1.2, we introduce a set of V2X applications as a representative of the connected vehicle vision. After that, we introduce the attributes used for characterizing those applications in Section 1.3. In Section 1.4, we characterize each application according to the introduced attributes; this constitutes a fundamental step towards identifying a few generic application classes. Next, we introduce the market perspectives and challenges for deployment for each representative application class in Section 1.5. Finally, we conclude the chapter and lay out potential directions for future research in Section 1.6.

\subsection{V2X Applications from the User Benefits Perspective}

Research on VANET technology has been driven mainly by the demand for the provision of network support for application development. So far, the DSRC research community has developed a large number of potential V2X applications for future deployment, ranging from safety/warning applications and highway traffic management to commercial applications. Since it is difficult to analyze a large number of applications, we chose 16 representative ones based on criteria such as customer value, near-term feasibility of deployment, technical novelty, and diversity of enabling technologies. The chosen applications (shown in Tables 1.1, 1.2 , and 1.3) constitute the basis for our study.

From a value or customer benefit perspective, these applications can be roughly organized into three major categories: safety-oriented, convenience-oriented, and commercial-oriented. These categories are derived from the characteristics and customer benefits of the applications. Note that, among those listed, safety-oriented applications are of special interest because they are expected to reduce the fatalities and economic losses caused by traffic crashes. 
- Safety-oriented applications (shown in Table 1.1) actively monitor the nearby environment (the state of other vehicles or road conditions) via message exchanges between vehicles, so that applications are able to assist drivers in handling the upcoming events or potential danger. Some applications may automatically take appropriate actions (such as automatic braking) to avoid potential crashes, while others provide only advisory or warning information as configured by the driver. The latter category of applications is very similar to the former, even though the system requirements (such as reliability, latency, etc.) are less stringent. However, both types of applications aim to improve the level of vehicle safety.

Table 1.1 V2X safety-oriented applications of interest

\begin{tabular}{|c|c|c|}
\hline Acronym & Name & Description \\
\hline SVA & $\begin{array}{l}\text { Stopped or } \\
\text { Slow Vehicle } \\
\text { Advisor }\end{array}$ & $\begin{array}{l}\text { A slow or stopped vehicle broadcasts warning messages to approaching } \\
\text { vehicles while it is slow/stopped. Approaching vehicles notify their drivers } \\
\text { of the slow/stopped vehicle. }\end{array}$ \\
\hline EEBL & $\begin{array}{l}\text { Emergency } \\
\text { Electronic } \\
\text { Brake Light }\end{array}$ & $\begin{array}{l}\text { A vehicle braking hard broadcasts warning messages to approaching } \\
\text { vehicles during the hard braking. Approaching vehicles notify their drivers } \\
\text { of the hard braking event. }\end{array}$ \\
\hline $\mathrm{PCN}$ & $\begin{array}{l}\text { V2V Post } \\
\text { Crash } \\
\text { Notification }\end{array}$ & $\begin{array}{l}\text { A vehicle involved in a crash broadcasts warning messages to } \\
\text { approaching vehicles until the crash site is cleared. Approaching vehicles } \\
\text { notify their drivers of the crash. }\end{array}$ \\
\hline $\mathrm{RHCN}$ & $\begin{array}{l}\text { Road Hazard } \\
\text { Condition } \\
\text { Notification }\end{array}$ & $\begin{array}{l}\text { A vehicle detecting a road hazard (e.g. a pothole or ice) broadcasts } \\
\text { warning messages to vehicles within the affected region. Approaching } \\
\text { vehicles notify their drivers of the hazard. }\end{array}$ \\
\hline RFN & $\begin{array}{l}\text { Road Feature } \\
\text { Notification }\end{array}$ & $\begin{array}{l}\text { A vehicle detecting an advisory road feature (e.g. sharp curve, steep } \\
\text { grade) broadcasts warning messages to approaching vehicles, which } \\
\text { notify their drivers of the advisory road feature. }\end{array}$ \\
\hline $\mathrm{CCW}$ & $\begin{array}{l}\text { Cooperative } \\
\text { Collision } \\
\text { Warning }\end{array}$ & $\begin{array}{l}\text { A vehicle actively monitors kinematics status messages that are } \\
\text { broadcast from other vehicles in its neighborhood to warn the driver of } \\
\text { potential collisions. }\end{array}$ \\
\hline CVW & $\begin{array}{l}\text { Cooperative } \\
\text { Violation } \\
\text { Warning }\end{array}$ & $\begin{array}{l}\text { A roadside unit actively broadcasts signal phase, timing and related } \\
\text { information to approaching vehicles. The vehicles use this information to } \\
\text { warn drivers of potential signal violations. }\end{array}$ \\
\hline
\end{tabular}

- Convenience-oriented (traffic management) applications (shown in Table 1.2) share traffic information among roadway infrastructure, vehicles on the road, and centralized traffic control systems, to enable more efficient traffic flow control and maximize vehicle throughput on the road. Ultimately, these applications not only enhance traffic efficiency, but also boost the degree of convenience for drivers.

- Commercial-oriented applications (shown in Table 1.3) provide drivers with various types of communication services to improve driver productivity, entertainment, and satisfaction, such as web access and streaming audio and video.

\subsubsection{Application value}

Driver assistance and safety features are capable of providing comprehensive information about the surrounding environment to drivers. For example, such features can inform drivers 
Table 1.2 V2X convenience-oriented applications of interest

\begin{tabular}{|c|c|c|}
\hline Acronym & Name & Description \\
\hline CRN & $\begin{array}{l}\text { Congested } \\
\text { Road } \\
\text { Notification }\end{array}$ & $\begin{array}{l}\text { A vehicle detects road congestion and broadcasts the information to } \\
\text { other vehicles in the region so that other vehicles can use the information } \\
\text { for alternate route and trip planning. }\end{array}$ \\
\hline TP & Traffic Probe & $\begin{array}{l}\text { A probe vehicle aggregates traffic probe information by actively } \\
\text { monitoring kinematics status messages that are broadcast from other } \\
\text { vehicles and transmits this information through roadside units to a traffic } \\
\text { management center. }\end{array}$ \\
\hline TOLL & $\begin{array}{l}\text { Free Flow } \\
\text { Tolling }\end{array}$ & $\begin{array}{l}\text { A vehicle entering a highway toll gate receives a beacon from the toll } \\
\text { gate's roadside unit. The vehicle establishes unicast communication with } \\
\text { the toll gate roadside unit and an E-payment transaction is completed for } \\
\text { toll payment. This enables roadway and congestion pricing via non-stop } \\
\text { tolling. }\end{array}$ \\
\hline PAN & $\begin{array}{l}\text { Parking } \\
\text { Availability } \\
\text { Notification }\end{array}$ & $\begin{array}{l}\text { A driver looking for an available parking facility drives the vehicle within } \\
\text { communication range of a Parking Availability Notification roadside unit. } \\
\text { The vehicle sends a request to the roadside unit for a list of nearby } \\
\text { parking locations, and the roadside unit responds with a list of parking } \\
\text { locations and availability. The vehicle may sort responses according to } \\
\text { distance from the current location, and presents a list showing available } \\
\text { parking lots within a certain geographical region to the driver. }\end{array}$ \\
\hline PSL & $\begin{array}{l}\text { Parking Spot } \\
\text { Locator }\end{array}$ & $\begin{array}{l}\text { A vehicle entering a parking area or structure sends a request to the } \\
\text { Parking Space Locator roadside unit for a list of open parking spaces. } \\
\text { The roadside unit sends a list of open spaces and an optional map to the } \\
\text { vehicle, and the vehicle notifies the driver of their locations. }\end{array}$ \\
\hline
\end{tabular}

of construction zones, speed limits, curves, and broken down vehicles well in advance. In terms of performance when compared to traditional sensors, communication-based safety features take advantage of the relatively long communication range provided by DSRC in order to sense distant events that occur outside the sensing range of traditional sensors. This advance notice can provide drivers with ample time to plan maneuvers around obstacles, or to plan changes in their route. Some aspects of communication-based features have already been described in terms of their safety value by VSCC (2005). The value of a system that relies entirely on safety communications can be bounded by the features implemented, the crash statistics pertaining to that feature, and the market penetration. For instance, a forward collision alert application can help to reduce rear-end crashes, which account for roughly $30 \%$ of all crashes in the USA each year. Vehicular communication can also enable numerous types of new applications. For example, since a radar sensor cannot see through objects, there is no way for it to provide an indication that a vehicle a few cars ahead has braked hard. An Emergency Electronic Brake Light (EEBL) feature has the potential to assist drivers of both equipped and unequipped vehicles by providing useful advance information and potentially reducing traffic shockwaves and pile-up accidents.

The value of convenience and traffic efficiency features has currently not been studied in much detail. Transportation efficiency features improve the customer's experience very indirectly by making the roadways and the organizations that maintain them more efficient. There are many individual services that can be provided by this set of features, and each may require a different value model. Traditional ETC systems have been shown to dramatically improve throughput on a per-lane basis when compared with manual toll collection techniques. In Japan it was found that $30 \%$ of roadway congestion was due to 
Table 1.3 V2X commercial-oriented applications of interest

\begin{tabular}{|c|c|c|}
\hline Acronym & Name & Description \\
\hline RVP/D & $\begin{array}{l}\text { Remote } \\
\text { Vehicle Per- } \\
\text { sonalization/ } \\
\text { Diagnostics }\end{array}$ & $\begin{array}{l}\text { When a vehicle is within communication range of the driver's home, the } \\
\text { driver may initiate a wireless connection of the vehicle to the home } \\
\text { network and download (or upload) the latest personalized vehicle } \\
\text { settings. This allows drivers to personalize their vehicle settings remotely. } \\
\text { Also, when a vehicle is within the service bay at a dealership, the driver } \\
\text { may initiate a wireless connection between the vehicle and the dealer } \\
\text { service network in order to upload the latest vehicle diagnostics } \\
\text { information and download any new updates. }\end{array}$ \\
\hline SA & $\begin{array}{l}\text { Service } \\
\text { Announcement }\end{array}$ & $\begin{array}{l}\text { Businesses (e.g. a fast food restaurant) may use roadside infrastructure } \\
\text { to announce services to vehicles wirelessly as they pass within } \\
\text { communication range. A vehicle may inform its driver of the services } \\
\text { based on the driver's subscription or request for such information. }\end{array}$ \\
\hline CMDD & $\begin{array}{l}\text { Content, Map } \\
\text { or Database } \\
\text { Download }\end{array}$ & $\begin{array}{l}\text { When a vehicle is within proximity of a home or hot-spot, the driver may } \\
\text { initiate and connect wirelessly to the home network or hot-spot so that } \\
\text { the vehicle can download content (e.g. maps, multimedia or web pages) } \\
\text { from the network to the hard drive radio/navigation system. }\end{array}$ \\
\hline RTVR & $\begin{array}{l}\text { Real-time } \\
\text { Video Relay }\end{array}$ & $\begin{array}{l}\text { A vehicle may initiate transmission of real-time video that may be useful } \\
\text { to other drivers in the area (e.g. a traffic jam scene). Other vehicles may } \\
\text { display this information to their drivers and also relay the real-time video } \\
\text { via multi-hop broadcasts in order to extend the range of the } \\
\text { communicated information to other vehicles or roadside units. }\end{array}$ \\
\hline
\end{tabular}

toll gates. A reduction in roadway congestion has a direct benefit to society in terms of fuel consumption and $\mathrm{CO}_{2}$ emissions. Additional benefits such as increased productivity and personal time may also be realized, though perhaps at a level that is not as noticeable to the customer.

Commercial features may be able to create substantial demand from consumers since they span many aspects of a customer's daily life. However, commercial features will in general require a strong non-OEM provider with necessary infrastructure support to enable such services. Some of the services and activities enabled by commercial features include news, traffic and weather updates, Internet and email access, music downloads, and drivethrough payments. Currently there has been little work performed that shows the value of these types of applications. Again, there are many individual services that can be provided through this set of features, and each may require a different value model. Commercial applications certainly have an opportunity to increase many aspects of customer convenience. In Japan, ETC systems based on DSRC have been used to make payments at parking garages. Electronic drive-through payment systems may be able to substantially increase vehicle throughput just as ETC systems have. By reducing the wait time at fast food restaurants, car washes or parking garages, applications should be able to benefit customers directly in terms of time saved. Likewise, there are many types of information-access services that could be provided to consumers in a personalized manner, something traditional radio services can currently not provide. For example, instead of the traditional broadcast news, a custom version of the news, based on customer preferences, could be downloaded from the customer's home to the car before a morning drive. 


\subsection{Application Characteristics and Network Attributes}

In this section, we define the application and networking criteria used in our classification. Careful selection of these criteria is critical to adequately capture the subtle, yet important, differences between various applications, and their diverse networking requirements. Thus, our approach was first to enumerate the characteristics of the applications in Tables 1.1, 1.2, and 1.3 in a systematic and thorough manner so that we could gain important insight into the applications, and then to use this insight to explore the demands these applications place on network design and enumerate their common network-related attributes. We group these criteria into two major divisions, application-related characteristics and network-related attributes, which are discussed in the next sections.

\subsubsection{Application characteristics}

In this section, we introduce the application-related characteristics that we identified and used as the basis for our proposed classification. These characteristics, summarized in Table 1.4, describe properties directly related to the applications themselves, such as user benefit and affected geographical region. As mentioned previously, the goal is to develop key characteristics that cover the various design aspects of the set of applications that we have explored. While we have attempted to be as general and as thorough as possible, we acknowledge that future analysis of a broader set may uncover other important characteristics. Indeed, it is our hope that the work presented here will inspire others to research and expand the list as future applications are explored and developed. However, as we will show, this list covers a sufficiently broad range to be a useful reference tool for application and network designers. In the remainder of this section we discuss these characteristics in more detail.

Table 1.4 Candidate criteria to characterize and classify applications (application characteristics)

\begin{tabular}{|c|c|c|}
\hline Application Characteristics & Description & Choices \\
\hline User benefit of application & $\begin{array}{l}\text { What benefit does the application } \\
\text { bring to users? }\end{array}$ & $\begin{array}{l}\text { Safety, Convenience, } \\
\text { Commercial }\end{array}$ \\
\hline Participants of application & $\begin{array}{l}\text { What entities participate in the } \\
\text { application? }\end{array}$ & V2V, V2I \\
\hline $\begin{array}{l}\text { Application } \\
\text { Region-of-Interest }\end{array}$ & $\begin{array}{l}\text { What is the size of the affected } \\
\text { geographical region of the application? }\end{array}$ & Long, Medium, Short \\
\hline $\begin{array}{l}\text { Application trigger } \\
\text { condition }\end{array}$ & $\begin{array}{l}\text { When and how is the application } \\
\text { triggered? }\end{array}$ & $\begin{array}{l}\text { Periodic, Event-driven, } \\
\text { User-initiated }\end{array}$ \\
\hline $\begin{array}{l}\text { Recipient pattern of } \\
\text { application message }\end{array}$ & $\begin{array}{l}\text { What is the pattern of recipients for } \\
\text { the application messages? }\end{array}$ & $\begin{array}{l}\text { One-to-one, One-to-many, } \\
\text { One-to-a-zone, Many-to-one }\end{array}$ \\
\hline Event lifetime & How long does the event last? & Long, Short \\
\hline Event correlation & $\begin{array}{l}\text { What is the degree of event correlation } \\
\text { in the Region-of-Interest? }\end{array}$ & Strong, Weak, None \\
\hline Event detector & $\begin{array}{l}\text { How many hosts can detect/generate } \\
\text { the event? }\end{array}$ & Single host, Multiple hosts \\
\hline
\end{tabular}


- User benefit describes the type of benefit or value the application provides to the end customer, as defined in a number of studies (VSCC 2005) (and discussed in Section 1.2). Overall, there are three widely accepted types: safety-oriented applications, convenience-oriented applications, and commercial-oriented applications.

- Application participants specifies the entities that may potentially be involved in the application. Some applications only require communication among vehicles, while others require coordination between vehicles and roadside infrastructure. Hence, communication-based automotive applications can be categorized as either $\mathrm{V} 2 \mathrm{~V}$ or V2I applications.

- Application Region-of-Interest (ROI) is the size of the geographical region covered by those entities participating in an application. Different kinds of applications have different ROI sizes. For example, in some safety applications, vehicles need to be aware of the kinematics status of other vehicles in their direct neighborhood (i.e. a few hundred meters), whereas in other safety applications vehicles need to know the hazard situation of a stretch of road that lies ahead (i.e. up to 1 kilometer). Likewise, for some convenience applications, vehicle occupants may want to know the status of road congestion far ahead (i.e. several kilometers) for trip planning. Qualitatively, the application ROI can be classified into three major types: short-, medium-, and long-range. Quantitative characterization of the shape and dimensions of the ROI, for various applications, is an important topic that requires interdisciplinary research in system reliability, driver behavior, and traffic/road dynamics to name a few.

- Application trigger condition specifies how applications are triggered. This is generally either periodic, event-driven, or user-initiated. Implicitly, it also specifies the kind of communication methods used by the application. For example, the vehicular kinematics status messages used for collision detection are normally broadcast periodically, whereas warning messages for events such as panic braking are usually event-driven, and request messages for on-demand convenience services from vehicle occupants are generally user-initiated.

- Recipient pattern of application message specifies the pattern of potential message recipients for an event, which varies across applications. For instance, for safety applications like Cooperative Collision Warning (CCW) and Cooperative Violation Warning (CVW), it is critical for all neighboring vehicles to hear broadcast safety alert messages to avoid potential collisions (a one-to-many pattern), whereas for safety applications such as EEBL, Stopped or Slow Vehicle Advisor (SVA), and Post Crash Notification (PCN), only vehicles in the region being affected (vehicles behind the event originator) need to hear the safety alert message (a one-to-a-zone pattern). Likewise, a point-to-point communication pattern is often used in many convenience and commercial applications, and a many-to-one pattern is also sometimes used. Thus, the pattern of event message recipients can be grouped into four categories: one-tomany, one-to-a-zone, one-to-one, and many-to-one.

- Event lifetime illustrates how long an application event (e.g. traffic crash or road congestion) persists over time. Among the criteria discussed so far, event duration is one application characteristic that may directly affect network system design. Among all applications, event lifetime may differ significantly. For example, some events have 
relatively short durations (e.g. EEBL events may last only a few seconds on average), while others may have relatively long durations (e.g. a PCN event may take hours before the crashed vehicles are cleared from the roadway). Among the applications we studied, most fell into one of two general categories: either a short event lifetime on the order of seconds or a long event lifetime on the order of minutes to hours.

- Event correlation specifies the degree to which events generated by entities within a geographical region of interest are correlated with each other. For example, the occurrence of an EEBL event in a vehicle may be highly correlated to EEBL events generated by other vehicles in front of it. Another example is Road Hazard Condition Notification (RHCN), where RHCN events in nearby vehicles may be highly correlated since they are caused by the same road hazard condition. Qualitatively, applications can be grouped into three categories: those with strong event correlation, weak event correlation, and no event correlation.

- Event detector specifies how many vehicles generate event messages in response to the same event. For instance, for applications such as SVA or PCN, where a vehicle reports its kinematics status, the vehicle is the sole event detector (i.e. of its kinematics state) and event message host (originator), whereas for applications such as RHCN and Road Feature Notification (RFN), where a vehicle reports road hazards, many vehicles may detect the same event (i.e. the same road hazard) and serve as event message hosts. Therefore, we classify application event detection as either single host or multiple hosts.

As mentioned previously, we believe these are the key defining characteristics, among the 16 applications that we studied, that are of most relevance to network design. However, we acknowledge that further application analysis may reveal other characteristics to add to the list, and we hope that it inspires others to do so. For the purposes of this study, however, these are the basis for the application characteristics portion of our classification effort. In the next section we present our group of key network-related attributes and their relation to the application characteristics above.

\subsubsection{Network attributes}

In this section we introduce the key network-related attributes that we used in our classification to characterize the fundamental aspects of network design for communication-based automotive applications. These attributes, summarized in Table 1.5, are somewhat related to the application characteristics discussed in the previous section, as we will show. In the remaining part of this section we discuss these network attributes, and their relationship with the corresponding application characteristics, in detail.

- Channel frequency specifies the type of physical-layer channels that may be used to support communication-based automotive applications. Following FCC regulations in the USA, safety-oriented applications are normally assumed to use a single DSRC-Control Channel $(\mathrm{CCH})$, whereas convenience-oriented applications use one of six DSRC-Service Channels (SCHs). On the other hand, commercial-oriented applications can either occupy DSRC-SCH channels, or any other channel frequency in the unlicensed Industrial, Scientific, and Medical (ISM) bands (e.g. Wi-Fi 2.4 and $5.8 \mathrm{GHz}$ ). In other words, the choice of channel is largely determined by the value of 
Table 1.5 Candidate criteria to characterize and classify applications (network attributes)

\begin{tabular}{|c|c|c|}
\hline Application Attributes & Description & Choices \\
\hline Channel frequency & $\begin{array}{l}\text { What channel does the application } \\
\text { use? }\end{array}$ & $\begin{array}{l}\text { DSRC-CCH, DSRC-SCH, } \\
\text { Wi-Fi }\end{array}$ \\
\hline Infrastructure & Is infrastructure required? & Yes, No \\
\hline Message time-to-live & How far do messages propagate? & Single-hop, Multi-hop \\
\hline Packet format & What type of packet is used? & WSMP, IP \\
\hline Routing protocol & How are messages distributed? & $\begin{array}{l}\text { Unicast, Broadcast, Geocast, } \\
\text { Aggregation }\end{array}$ \\
\hline $\begin{array}{l}\text { Network protocol initiation } \\
\text { mode }\end{array}$ & How is a network protocol initiated? & $\begin{array}{l}\text { Beacon, On-demand, } \\
\text { Event-triggered }\end{array}$ \\
\hline Transport protocol & $\begin{array}{l}\text { What form of end-to-end } \\
\text { communication is needed? }\end{array}$ & $\begin{array}{l}\text { Connectionless, } \\
\text { Connection-oriented }\end{array}$ \\
\hline Security & What kind of security is needed? & $\begin{array}{l}\text { V2V security, V2I security, } \\
\text { Internet security }\end{array}$ \\
\hline
\end{tabular}

the user benefit characteristic of the application. While there are many other channels that can be used (such as cellular telephony or WiMAX), in practice the choice of channel is generally one of either $\mathrm{DSRC}-\mathrm{CCH}, \mathrm{DSRC}-\mathrm{SCH}$, or Wi-Fi. In other regions of the world, a similar choice needs to be made for the applications to be effective.

- Infrastructure specifies whether the application needs infrastructure (i.e. a roadside unit) for its operation. Obviously, this is needed if the participants of the application characteristic involves a roadside unit. Otherwise, it may not be required.

- Message Time-to-Live (TTL) specifies how far a message is propagated by the network, and what type of packet forwarding/routing functionality (i.e. single-hop or multihop) is needed by the network layer. This attribute is partly determined by the application region of interest characteristic. Single-hop communication is sufficient for short-range applications, while medium- or long-range applications require multihop packet forwarding/routing functionality for extended reachability. Thus, design choices include either single-hop or multi-hop routing.

- Message packet format describes the format of the network packets that are used to encapsulate the application messages. This attribute is partly influenced by the user benefit characteristic of the application. In general, the automotive industry (VSCC 2005) and the IEEE standard community (IEEE 802.11 1999) have promoted the idea that safety and convenience applications are more likely to use relatively constant and small-sized packets, whereas commercial applications are more likely to use variable and large-sized packets. In the DSRC standard developed in the USA, the WAVE Short Message Protocol (WSMP) is proposed for safety and convenience use. It is essentially a simplified version of the IP protocol, with a smaller packet header to reduce perpacket overhead for improved network efficiency. For commercial applications, it is assumed that the traditional IP packet format will be used. Thus, we classify packet formats into two types: either WSMP format or IP format.

- Routing protocol is a design choice that illustrates what kind of network routing protocols are used for the various applications. Obviously, this network attribute is 
closely related to the recipient pattern of application message characteristic. For instance, most safety applications use broadcast routing (one-to-many) or geocast routing (one-to-a-zone), while convenience and commercial applications normally use unicast routing (one-to-one) or aggregation routing (many-to-one).

- Network protocol initiation mode describes how the network protocol is triggered. Some safety applications mandate periodic broadcast 'beaconing' of status messages, like CCW and CVW (i.e. beacon mode), whereas other safety applications, like EEBL and PCN, send messages only when a critical event is detected (i.e. event-triggered mode). For a portion of convenience and commercial applications, it is the vehicle occupants that initiate message announcements and service request (i.e. user-initiated on-demand mode).

- Transport protocol is a design choice that indicates whether or not a reliable end-toend connection is needed to support the application. As we discovered, safety and convenience applications generally follow the connectionless paradigm (e.g. WSMP, UDP), while commercial applications often use the traditional connection-oriented paradigm (e.g. TCP).

- Security considers what kind of security solution is needed for the application. The choices include V2V security, V2I security and Internet security. Safety applications require high-level $\mathrm{V} 2 \mathrm{~V}$ security preventing vehicles from malicious attacks, convenience applications also mandate the stringent V2I security solution because financial transactions could be involved at roadside infrastructure, and most commercial applications require efficient collaboration between V2X security solutions and existing security solutions for the Internet.

As indicated earlier, many of these network attributes are closely related to specific application characteristics. Intuitively, a given application characteristic or performance requirement normally requires a given networking mechanism or capability. In the next section we show how sets of applications with similar characteristics and requirements lead to the same network solutions, resulting in a very useful and intuitive general classification.

\subsection{Application Classification and Categorization}

In this section we present the results of characterizing and classifying the set of 16 applications introduced in Section 1.2. We then compare and contrast these applications, first with respect to the application characteristics presented in Section 1.3.1, and then with respect to the network attributes presented in Section 1.3.2. Afterwards, we show how, by combining the applications with similar characteristics and network functionalities, we can group these applications into seven generic classes (from the perspective of network design).

\subsubsection{Characterization based on application characteristics}

The process of application characterization is divided into two steps: characterization of application attributes and characterization of network attributes (i.e. network design), as shown in Tables 1.6 and 1.7 respectively. By first exploring all the relevant application characteristics for each one, we gain a more complete understanding of the fundamental 
properties and functionality requirements of these applications. Later, we show how this effort gives rise to application characterization from the network design point of view.

Table 1.6 lays out the main characteristics of each application based on the selected application-related attributes summarized in Section 1.3.1. Given the limited space, we are unable to discuss the characteristics of all 16 applications. Instead, we only highlight a few important application characteristics, illustrating how these criteria help to differentiate the often subtle differences between various applications, as follows.

- Notice that most of the safety applications have a medium-sized effective application range (i.e. a few hundred meters to 1 kilometer), since safety messages, such as vehicular kinematics status or road conditions, are only relevant to other vehicles within a moderate geographical region. Exceptions are the CCW and CVW applications, which have a small application effective range because they require closer monitoring of vehicles in their direct neighborhood (i.e. within 200 meters). Conversely, convenience applications generally require a medium to large effective range (i.e. up to a few kilometers), because it is vital for drivers to know the congestion situation or traffic conditions at this range for effective detour or trip planning decision making. Similarly, commercial applications tend to have a large effective range in order to access remote commercial service providers. For example, a fast food restaurant is willing to announce its service to vehicles a long distance (i.e. several kilometers) from its location.

- Most safety applications (e.g. EEBL, RHCN and SVA) and few convenience applications (e.g. Congested Road Notification, or CRN, Traffic Probe, or TP, and Free Flow Tolling, or TOLL) are initiated by the events happening on the road, such as vehicle collisions, detection of road hazards (e.g. ice or oil), sudden braking, or detection of traffic congestion. If no such events happen, these applications will not be called upon. Among safety applications, CCW and CVW are unusual because they rely on the periodic message updates to monitor the neighboring vehicles' driving status, regardless of safety events. On the other hand, most convenience and commercial applications are triggered on demand by vehicle occupants, rather than by any safety event on the road or the vehicle itself.

- The potential recipients of application messages, in most safety applications (e.g. SVA and EEBL), are vehicles within a specific zone (i.e. behind the vehicle that detects the event and originates the safety message). Thus, safety applications can be summarized as having one-to-a-zone recipient patterns. Again, CCW and CVW do not follow this general trend. In these two applications, all the vehicles in the neighborhood are supposed to receive the periodic update in order to avoid the potential crash from any direction. So these two applications have one-to-many recipient patterns. At the same time, convenience and commercial applications vary from application to application: some convenience applications (e.g. TOLL) and commercial applications (e.g. Remote Vehicle Personalization/Diagnostics, or RVP/D, Content, Map or Database Download, or CMDD, and Real-time Video Relay, or RTVR) belong to the point-to-point (one-toone) communication paradigm, while other convenience (e.g. CRN) and commercial applications (e.g. Service Announcements, or SAs) are fundamentally one-to-a-zone in nature. 


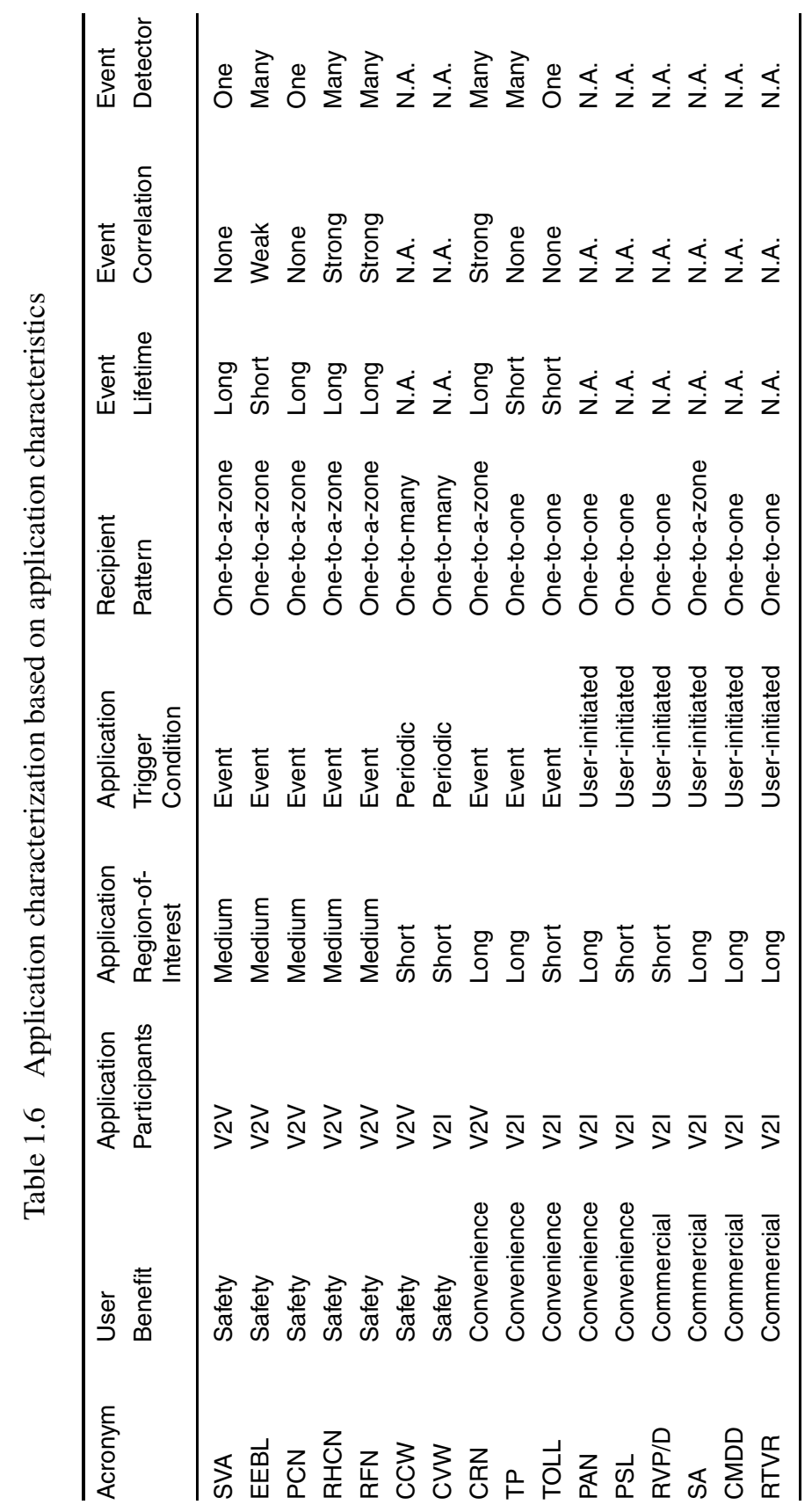


- 'Event' is an important concept in safety applications, and inxs a few convenience applications, because it is an event that initiates the application operations. In our study we also characterize safety events via several properties, including event duration, event correlation, and event detectors. Consistent with our conjecture, we find that safety events drastically vary from application to application. For example, sudden braking (EEBL) is a one-shot event, while road hazard/feature events (RHCN or RFN) are persistent ones. Also, different instances of RHCN or RFN events caused by the same road hazard/feature are more likely to be correlated with each other, in contrast with the totally independent PCN events. Even though the study of event characteristics is not directly used in the network design conducted in Section 1.4.2, we believe that such an analysis can assist future network designers better to capture the data traffic patterns induced by event-driven safety applications.

From an application benefits point of view, different applications have different functionalities, providing different usages for customers. Interestingly enough, we realize that many applications exhibit highly similar application characteristics, with the exception of a few minor differences. To validate whether such an observation is valid from a network design perspective, we conduct an application characterization based on the relevant network attributes in Section 1.4.2.

\subsubsection{Characterization based on network attributes}

As mentioned in Section 1.3.2, for each application we discovered that its characteristics tend to mandate a certain design in the network protocol stack. For example, applications with oneto-many recipient patterns are more likely to use broadcast routing protocols, while unicast routing protocols are suitable for applications with one-to-one recipient patterns. Similarly, a single-hop packet dissemination mechanism is adequate to support applications with small application ROIs (i.e. a few hundred meters). In contrast, multi-hop routing protocols are needed for applications with medium or large application ROI. Accordingly, we are capable of determining the potential design choices for various components in the network stack by referring to their corresponding application characteristics and requirements. At the same time, we also notice that some of the network attributes are purely the choices of network designers, since different technical approaches are able to achieve the same objective.

Table 1.7 lays out the main network attributes of each application based on the selected network attributes summarized in Section 1.3.2, from the lower physical layer to the upper transport layer. These network attributes cover design issues such as the physical layer channel frequency, the usage of infrastructure, message TTL, routing protocol and network protocol triggers at the network layer, transport layer design, and security solutions. Again, we emphasize only a few important network attributes, discussing the potential impact of application characteristics on these network design issues.

- The message packet format is determined by the type of application (from the perspective of user benefit). Normally, safety and convenience applications use lightweight short messages in the WSMP format, to improve network resource efficiency. Commercial applications, on the other hand, generally prefer the traditional heavyweight IP format to be compatible with existing Internet commercial services.

- The network-layer routing protocol is one essential component in a network stack, differentiating the reachability and recipient patterns of various applications. 


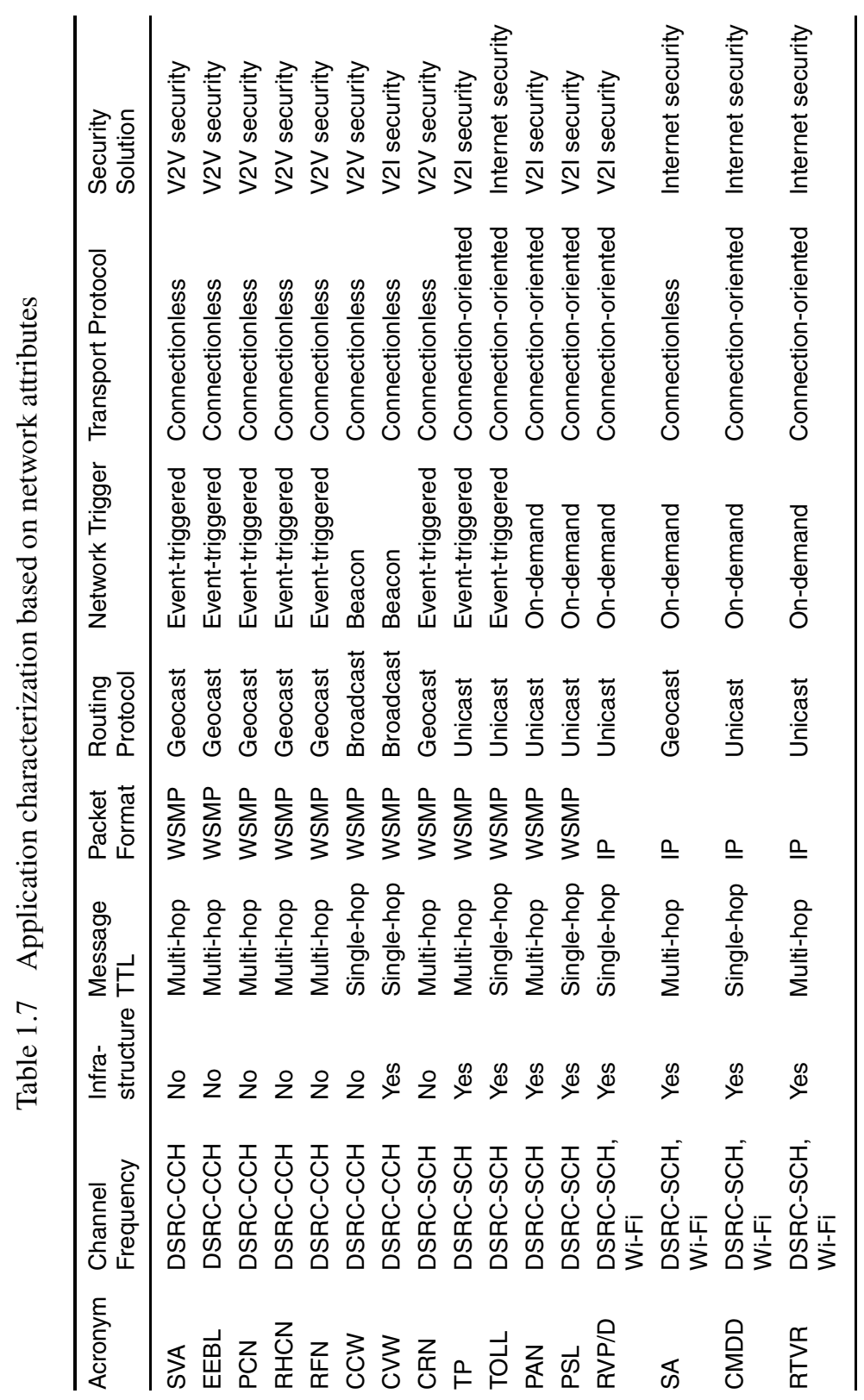


Most safety applications utilize multi-hop geocast routing protocols, because of the one-to-many communication nature of safety applications. Geocast routing distributes packets within a given zone or region. Thus, a geocast routing protocol can be viewed as a special case of broadcast routing. CCW and CVW applications, instead, use the single-hop broadcast scheme to announce the periodic update in their direct neighborhood. Convenience and commercial applications either use geocast/broadcast protocols to announce messages in a region (for advertisement services such as SAs, or traffic congestion notifications such as $\mathrm{CRN}$ ), or exploit unicast protocols to forward packets to a given destination (for financial transactions like TOLL, or data downloads from infrastructure as with CMDD).

- How the network routing protocol is triggered is another interesting design choice to be examined in our study. Event-driven safety applications (e.g. SVA, EEBL, and CRN) require the event-triggered mechanism in network protocols, periodic-based safety applications (e.g. CCW and CVW) mandate the periodic beacon (or 'hello message') mechanism, and user-initiated convenience and commercial applications (e.g. SAs, RVP/D, and Parking Spot Locator, or PSL) are triggered in an on-demand fashion.

- The involvement of infrastructure in network design and application development is another key issue for consideration. Please note that the term 'infrastructure' in this chapter only refers to roadside units along the roads, rather than base stations in cellular systems. Two minor differences exist between infrastructure and vehicles: (a) infrastructure is stationary while vehicles are mobile; (b) infrastructure may have a direct connection to the Internet, but vehicles do not. Both infrastructureoriented approaches and non-infrastructure approaches (or even a combination of both approaches) are used to achieve the objective of supporting the applications or services discussed above. Deployment of infrastructure-oriented services depends on considerations such as availability of infrastructure, costs, and technology. The availability of infrastructure would facilitate the design and deployment of convenience applications. Having infrastructure that provides a gateway to the Internet would enable the design and deployment of commercial applications. As a side note, the involvement of infrastructure also complicates the design of security solutions. We believe that security solutions for $\mathrm{V} 2 \mathrm{~V}$ applications are different from those for V2I applications. Also, the gateway to the Internet requires compatibility of V2X security solutions with the existing Internet security solutions.

Throughout our study we found that Tables 1.6 and 1.7 reveal a number of interesting observations. Generally speaking, many applications exhibit highly similar application characteristics, resulting in similar protocol design across various layers in the network stack. For instance: (a) RHCN and RFN are nearly the same, except that the type of safety warning messages are different - RHCN is about road hazards, while RFN is about road features; (b) PCN and RHCN are also similar except for the number of event originators - PCN has a sole message host, while RHCN has multiple message hosts - and even though this difference gives rise to different levels of data traffic burstiness from event generation, the network protocol stacks for these two applications are still similar to each other; (c) CCW and CVW applications can be categorized into the same type, although the former is a V2V application whereas the latter is a V2I one.

In summary, the first seven safety applications (SVA, EEBL, PCN, RHCN, RFN, $\mathrm{CCW}$, and CVW) all utilize broadcast/geocast routing protocols to distribute safety/warning 
messages in the WSMP format. On the other hand, some convenience applications mostly rely on user-initiated unicast routing protocols to deliver non-safety messages in the WSMP format, while commercial applications may exploit IP protocols to enable enhanced functionality such as Quality of Service (QoS) routing. This, in turn, suggests that the studied applications naturally lend themselves to smaller numbers of generic/abstract classes, and this is the subject matter of the next section.

\subsubsection{Application classification}

With a deep understanding of application characteristics and network attributes applied to all studied applications, we are able to classify them into a number of generic classes. Notice that application classification can be conducted at different levels, depending on the design granularities. For example, simple classification and few abstract classes are adequate for high-level concept design of automotive communication applications. On the other hand, empirical design of prototype systems normally mandates an exhaustive effort, resulting in sophisticated multi-level application categories.

At the initial stage of this emergent research field, we believe that a high-level classification is sufficient to serve the purpose of distilling the major concepts and identifying the synergy among various applications, without unnecessarily complicating the problem formulation. Later on, the empirical prototype system can be designed and implemented based on the refined and enriched version of this study. Here we present such a way of classifying the aforementioned applications from the perspective of network design (as shown in Figure 1.1), among other alternatives. Generally speaking, V2X applications can be classified into two broad generic classes, namely short message communications and largevolume content download/streaming. Most safety and convenience applications belong to the first class, since the messages in these applications are lightweight WSMP messages. Considering that the IP message format is appropriate for large-volume data (such as Internet web access or video/audio streaming), most commercial applications fall into the second category.

\section{Short message communication}

First we discuss the class of short message communication, which uses lightweight WSMP packets. This class can be divided, depending on the recipient pattern and routing protocol, into either broadcast/geocast or unicast applications. Clearly, most safety applications require message announcements be sent to a large number of nodes (one-to-many or one-to-a-zone); hence they would fall into the broadcast/geocast-oriented type. On the other hand, many convenience applications (including payment-type applications) would fall into the unicastoriented type.

According to the network protocol triggering condition, broadcast/geocast-oriented applications can be further classified as event-driven, scheduled (periodic) and on-demand approaches. The event-driven class is used to model safety applications focusing on lifethreatening events, and the scheduled class is suitable for safety applications requiring periodic message updates, whereas the on-demand class is appropriate for convenience applications such as a parking spot locator. As a side note, high-level V2V security solutions are required to protect safety applications from malicious hackers. These three sub-classes of broadcast/geocast-oriented applications are: 


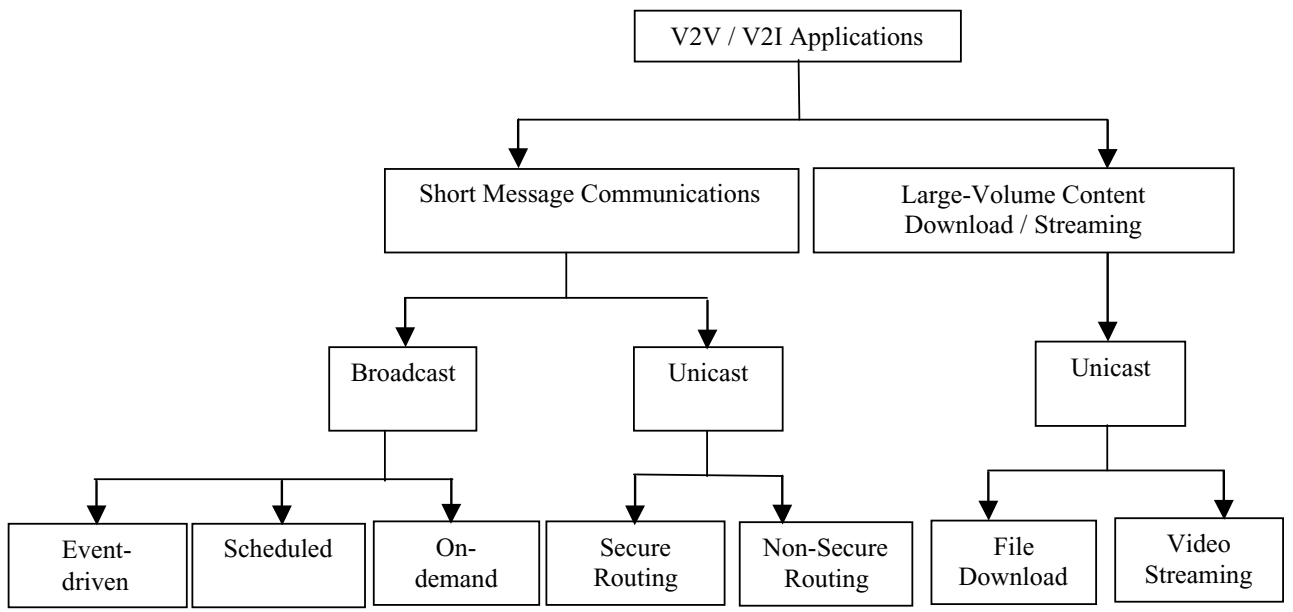

Figure 1.1 Classification from the perspective of network design

- Event-driven broadcast/geocast class: SVA, EEBL, PCN, RHCN and RFN applications, as well as CRN application, belong to this category (class 1).

- Scheduled (periodic) broadcast/geocast class: CCW and CVW applications fall into this category (class 2).

- On-demand broadcast/geocast class: Some convenience or commercial applications, such as SAs, belong to this category (class 3).

The secure routing of financial transactions in convenience applications also plays an important role in unicast-oriented applications. Thus, these unicast-oriented applications can be divided into those that involve stringent secure routing for financial transactions, and those that do not involve secure routing. Thus we list these two sub-classes of unicast-oriented applications:

- Secure unicast class: One example of this approach is a TOLL application (e.g. drivethrough payment or free-flow tolling). RVP/D also falls into this category since it is potentially related to the control components of vehicles (class 4).

- Unsecured unicast class: TP, Parking Availability Notification (PAN) and PSL applications fall into this category. Some commercial applications (e.g. V2V online chatting or social networking applications) also belong to it (class 5).

\section{Large-volume content download/streaming}

Next we focus on the second major class of applications, namely large-volume content download/streaming, which is normally implemented in the IP format for compatibility. These applications often utilize unicast protocols because of their one-to-one communication nature. This class is further divided depending on the content type: either file download or media streaming. The former type allows short-term disruption in network service, so it is inherently latency-tolerant. The latter type requires a relatively smooth streaming transfer, 
so it is fundamentally latency-sensitive. It is straightforward to notice the types of some large-volume content download/streaming applications:

- File download: a CMDD application (e.g. map database download or web access/ browsing) is one example of this approach (class 6).

- Video continuous streaming: RTVR (e.g. video/MP3 streaming among vehicles, or from roadside infrastructure for entertainment) falls into this category (class 7).

These seven types of V2X applications and their key considerations in network design are summarized in Table 1.8. From the above discussion, we conclude that the given set of applications can be grouped into seven generic classes. Since these applications are carefully chosen to represent many others, we believe that our classification methodology and its results can also apply to a large number of V2X applications.

Table 1.8 Network design considerations for seven types of applications

\begin{tabular}{|c|c|c|c|c|c|c|}
\hline $\begin{array}{l}\text { Application } \\
\text { Type }\end{array}$ & $\begin{array}{l}\text { Channel } \\
\text { Frequency }\end{array}$ & $\begin{array}{l}\text { Packet } \\
\text { Format }\end{array}$ & Routing Protocol & $\begin{array}{l}\text { Inter- } \\
\text { net }\end{array}$ & $\begin{array}{l}\text { Transport } \\
\text { Protocol }\end{array}$ & Security \\
\hline $\begin{array}{l}\text { Event-driven } \\
\text { broadcast/ } \\
\text { geocast }\end{array}$ & DSRC-CCH & WSMP & $\begin{array}{l}\text { Event-driven } \\
\text { multi-hop } \\
\text { broadcast/geocast }\end{array}$ & No & $\begin{array}{l}\text { Connection- } \\
\text { less }\end{array}$ & $\begin{array}{l}\text { V2V } \\
\text { security }\end{array}$ \\
\hline $\begin{array}{l}\text { Scheduled } \\
\text { broadcast/ } \\
\text { geocast }\end{array}$ & DSRC-CCH & WSMP & $\begin{array}{l}\text { Scheduled multi-hop } \\
\text { broadcast/geocast }\end{array}$ & No & $\begin{array}{l}\text { Connection- } \\
\text { less }\end{array}$ & $\begin{array}{l}\text { V2V/V2I } \\
\text { security }\end{array}$ \\
\hline $\begin{array}{l}\text { On-demand } \\
\text { broadcast/ } \\
\text { geocast }\end{array}$ & $\begin{array}{l}\text { DSRC-SCH, } \\
\text { or Wi-Fi }\end{array}$ & $\begin{array}{l}\text { WSMP, } \\
\text { or IP }\end{array}$ & $\begin{array}{l}\text { User-initiated } \\
\text { on-demand } \\
\text { multi-hop } \\
\text { broadcast/geocast }\end{array}$ & No & $\begin{array}{l}\text { Connection- } \\
\text { less }\end{array}$ & $\begin{array}{l}\text { V2V/V2I } \\
\text { security }\end{array}$ \\
\hline $\begin{array}{l}\text { Secure } \\
\text { unicast }\end{array}$ & DSRC-SCH & WSMP & $\begin{array}{l}\text { Multi-hop unicast } \\
\text { with secure routing }\end{array}$ & No & $\begin{array}{l}\text { Connection- } \\
\text { oriented }\end{array}$ & $\begin{array}{l}\text { Stringent } \\
\text { V2V/V2I } \\
\text { security }\end{array}$ \\
\hline $\begin{array}{l}\text { Normal } \\
\text { unicast }\end{array}$ & DSRC-SCH & WSMP & Multi-hop unicast & No & $\begin{array}{l}\text { Connection- } \\
\text { oriented }\end{array}$ & $\begin{array}{l}\text { V2V/V2I } \\
\text { security }\end{array}$ \\
\hline $\begin{array}{l}\text { File } \\
\text { download }\end{array}$ & $\begin{array}{l}\text { DSRC-SCH, } \\
\text { or Wi-Fi }\end{array}$ & IP & Multi-hop unicast & Yes & $\begin{array}{l}\text { Connection- } \\
\text { oriented }\end{array}$ & $\begin{array}{l}\text { V2V/V2I/ } \\
\text { Internet } \\
\text { security }\end{array}$ \\
\hline $\begin{array}{l}\text { Media } \\
\text { streaming }\end{array}$ & $\begin{array}{l}\text { DSRC-SCH, } \\
\text { or Wi-Fi }\end{array}$ & IP & $\begin{array}{l}\text { Single-hop unicast } \\
\text { with QoS routing }\end{array}$ & Yes & $\begin{array}{l}\text { Connection- } \\
\text { oriented }\end{array}$ & $\begin{array}{l}\text { V2V/V2I/ } \\
\text { Internet } \\
\text { security }\end{array}$ \\
\hline
\end{tabular}

The potential benefits of application classification are:

- The classification effort not only contributes to capturing the common features and technical requirements of applications, but also helps to develop common networking stacks for the identified generic classes. In the near future, with the deeper understanding of these generic and abstract classes, we shall be able to increase the module reusability of wireless networking solutions for the given set of applications with similar characteristics. 
- The classification effort helps to identify common requirements and performance metrics relevant to each application class. It also eases application modeling in simulation studies targeted at the performance evaluation of a large number of applications. By appropriately isolating generic network design from different application instantiations, we argue that it is much more efficient to model these seven generic classes than it is to model all 16 applications in an exhaustive manner without exploiting their noticeable commonality. Thus, a generic model should suffice for gathering statistics for the performance metrics defined for a specific class. Gathering performance results for a particular application, for the purposes of detailed analysis, could be achieved by deriving the application of interest as a simple extension from its generic model.

\subsection{Market Perspectives and Challenges for Deployment}

\subsubsection{Fleet penetration}

According to the DOT, Federal Highway Administration, there were a total of 237 million registered vehicles in the USA in 2004. Since the effectiveness of communication-based applications will require a certain level of market penetration, it will be a challenge to deploy a large number of equipped vehicles in a short time frame. Using production information from vehicle manufacturers, it is possible to project the length of time it will take for a sufficient level of market penetration to be achieved. A simple projection was performed using the figure of 237 million registered vehicles, together with the total number of vehicles produced by selected automobile manufacturers for 2005. It was assumed that the number of total vehicle registrations as well as the market share of the selected manufacturers will remain constant over time. The projection also assumes that every vehicle produced by the selected maker will be equipped with the V2X system.

As Figure 1.2 indicates, partnering strategies between automobile makers can result in an increased market penetration over a shorter period of time. At the very beginning of deployment, manufacturers will be adding technology to vehicles even though it has no immediate value to consumers. For this reason, it is likely that some kind of partnering strategy will emerge so that the features enabled by V2X become effective as soon as possible.

\subsubsection{System rollout options}

There are various options when it comes to the deployment of V2X systems.

- Standalone system solution: In a standalone implementation of an in-vehicle V2X system, we envision an additional embedded module, which executes the V2X functionality, added to the vehicle. This requires a processor with sufficient computation resources, a GPS receiver and wireless transceiver, vehicle network access, and, optionally, a human-machine interface. This is the most expensive approach, since the complete in-vehicle system infrastructure has to be added to execute the V2X functionality. The average cost would depend on the vehicle manufacturer; however, it should be clear that this option would be costly. 


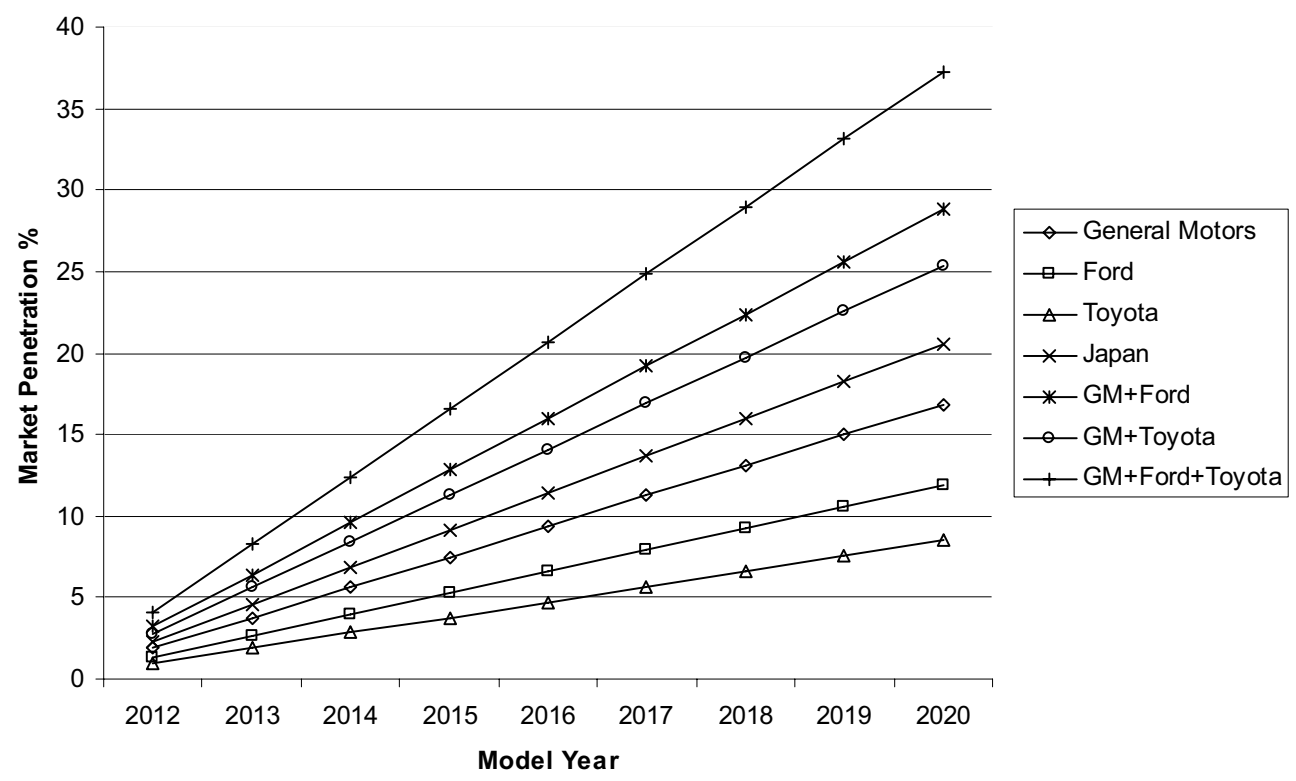

Figure 1.2 Projected US market penetration level of equipped vehicles (best case)

- Navigation system solution: Navigation systems will provide sufficient computing capabilities, since they are equipped with large amounts of memory, vehicle network access, and GPS. In terms of hardware, only the wireless radio unit and the antenna have to be added. In terms of software, the applications have to be added and the communication protocol has to be enabled (including security). However, growth of V2X would be directly proportional to and limited by the growth of navigation systems. In 2006 it was expected that there were between 1.5 million and 2 million in-vehicle navigation systems shipped in North America (ABI Research 2009). By 2011 this number is expected to climb substantially. Conservative estimates place the total at around 6.5 million, but it is possible that the volume of in-vehicle navigation systems could reach nearly 9 million by 2011 (ABI Research 2009). This corresponds to an overall fleet penetration of less than $4 \%$.

- Telematics system solution: This option is similar to the navigation system solution in that the system architecture required for V2X already exists for the most part. In terms of hardware, only the radio unit and the antenna have to be added. In terms of software, the applications have to be added and the communication protocol including security has to be enabled. The advantage of the telematics system solution is that the number of installed telematics units is much larger than the number of navigation systems. Telematics service provider OnStar currently has a total of 4 million subscribers, and for the 2008 model year OnStar systems will be standard on 50 out of 54 vehicles produced by General Motors. The OnStar service is also available for Acura and Lexus vehicles. Independent telematics service provider ATX currently has a total of 700,000 subscribers. ATX customers currently include Mercedes (Tele Aid) and BMW (Assist). 
- Aftermarket transceiver: This option is an aftermarket solution that enables older vehicles to function as communicating ones. The aftermarket transceiver solution has two potential types of embodiment: a passive version, and an integrated one that is capable of interfacing with the vehicle system in order to provide customers with a complete add-on solution. In the passive solution, everything required to support V2X communication is supplied on a device that reports GPS information to listening vehicles. However, consumers of a standalone transceiver do not receive any direct benefit from the device, other than making their vehicle visible to other equipped vehicles, since no information is displayed back to the customer. In the integrated solution, the device is capable of providing customers with a fully featured V2X system. In this type of implementation, a device that connects to the vehicle onboard diagnostic connector could interface with the GPS system of the vehicle and activate vehicle displays or audible chimes. An example of an integrated aftermarket transceiver would be a small device that plugs into the On-Board Diagnostic systems (OBDII) connector of the vehicle and provides advisory information to drivers through a Driver Information Center (DIC).

\subsubsection{Market penetration analysis}

Currently there is no common view about how to deploy communication technology economically in vehicles along with a suitable market introduction strategy to overcome the problems of the initial market penetration that many of the applications require. Communication-based systems are directly subjected to network effects (i.e. the value of this technology to the customer will increase as market penetration increases). In particular, for a $\mathrm{V} 2 \mathrm{~V}$ system, the customer will only perceive value once there is sufficient QoS provided by a minimum market penetration. Different application features will require different thresholds in terms of the minimum market penetration in order for this technology to become useful to consumers. Deployment decisions in all cases will be based on market penetration to ensure that customers receive a quantifiable benefit from the deployed applications. In all situations, the perceivable benefit to the consumers will increase as the market penetration level increases. With this in mind, the initial features to be deployed will be simple applications with very low market penetration level requirements. This deployment strategy will also allow us to gain valuable learning experience and help build customer confidence in the system and applications. The estimated minimum market penetration levels for the different broad application families defined in Section 1.2 will now be discussed. These estimates are based on preliminary research into this area and on engineering judgments about the penetration levels at which features become useful to consumers.

\section{Convenience (traffic management) and commercial applications}

The minimum required market penetration required for such applications is estimated to be very low. Communications infrastructure would serve as a catalyst for these applications and therefore the benefit to the vehicle customer would be seen even with low market penetration. While this may suggest that these types of applications might appear first, there must also be a roadway infrastructure available that provides services to the vehicles. Assuming such an infrastructure exists, some features will be able to provide immediate benefits to consumers, since the vehicle and the infrastructure will be able to communicate. Certain convenience 
(traffic efficiency) features may require a minimum penetration before there is sufficient collected data from equipped vehicles to make traffic management features viable. The latency of the information received from and sent to the vehicles could be relatively large without compromising the reliability of the feature.

\section{Safety applications}

For safety applications providing advisory information, it is expected that the driver would perceive value from the technology beginning at low market penetration levels. While these numbers seem promising, benefits of these types of features need to be studied to determine their actual and perceived value to consumers in terms of safety and convenience. It is suggested that simulation studies be used to measure the benefits of these types of applications in terms of crash reductions and increased traffic efficiency due to the reduction in crashes. The low minimum market penetration for these types of advisory features is primarily due to the fact that these features are uniquely enabled by communication technology and offer added value to the driving experience at a very low cost. However, while drivers would receive information they would not otherwise have, they may question the reliability or usefulness of a system that appears to function so infrequently. For these features to have value, a minimum system penetration would be required so that advisory information generated by an equipped vehicle is relayed to other vehicles often enough that consumers appreciate the benefit of it. If application information needs to be distributed over a large distance, an intelligent store and multi-hop message-forwarding scheme may be required.

For safety applications providing warning information, a higher estimated minimum market penetration level is required. Communication technology is not an enabler for these features since autonomous object detection sensors would achieve this objective and provide significant reliability to the driver. However, a collection of autonomous object detection sensors offering these warning features would be very expensive (since multiple sensors would be needed) and therefore autonomous sensors cannot be widely deployed in every vehicle segment. The trend shows that the market penetration of object detection sensors is extremely low and they can only be targeted for luxury vehicle segments. Thus it could be argued that since V2X technology is capable of providing these features at significantly low cost based on a single sensor, a majority of the drivers would benefit from these features at the market penetration levels indicated, and the system would provide more reliability as the market penetration increases. The societal benefits of transportation safety would be very significant even at modest penetration levels. Because current trends show very low market penetration levels of warning systems based on autonomous sensing, V2X may be able to provide a low cost technology alternative in a society that demands a significant reduction in annual vehicle crashes.

For safety features, it will be challenging to identify the market penetration levels at which drivers begin to perceive that the system is providing them with useful warnings. In terms of societal benefits, however, for an equipped vehicle pair that is involved in a potential collision there should be measurable reductions in vehicle crashes, property damage and personal injury. Collision avoidance control features would naturally be introduced only when the system is highly reliable, which would imply a very large market penetration. Since the requirement for fleet penetration is extremely high, it is likely these types of applications 
will only become available in the near term if the government mandates the technology and system, and all unequipped vehicles are also retrofitted.

\subsubsection{System rollout}

Based on the maturity of wireless technology, the advancements in positioning technology and optimistic market penetration forecasts, it is anticipated that the first V2X applications could reach the market by 2012. Further development of the V2X technologies as well as even further increases in fleet penetration levels will enable V2X crash warning features. The evolution of V2X feature deployment with respect to technology advancement and fleet penetration is shown in Figure 1.3.

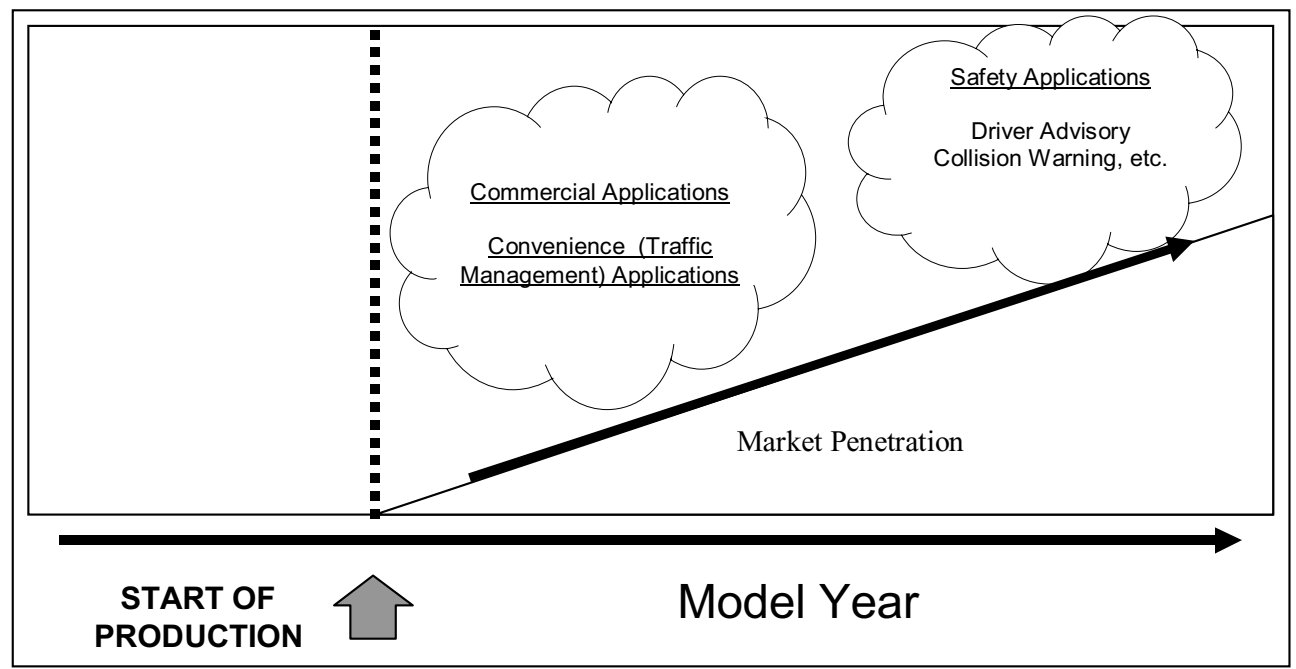

Figure 1.3 Evolution of V2V features with respect to fleet penetration

\subsubsection{Role of infrastructure}

Applications that can interface with infrastructure elements such as instrumented intersections or roadside units will be deployed as these structures become available. Infrastructurebased features are appealing to automakers since customers can potentially derive immediate benefits, given the availability of infrastructure hardware. Thus, in the deployment of V2I features, the only market penetration problem that needs to be solved is the deployment of the infrastructure hardware itself. Given that the number of hardware units required to support V2I features is much less than the number of communicating vehicles required to support V2V features, this proposition seems quite favorable. However, the deployment and maintenance of infrastructure hardware requires initiatives by government authorities or the establishment of agreements between automakers and private entities. The nature of these interactions can make it difficult to anticipate the availability of these services. This is 
in contrast with the deployment of $\mathrm{V} 2 \mathrm{~V}$ features, where automakers have control over the availability and timing of deployed systems.

The Vehicle Infrastructure Integration (VII) is a US government-sponsored activity that is oriented toward the nationwide deployment of DSRC-based communication infrastructure. This effort is leading toward a proof of concept demonstration and will ultimately lead to a field operational test. While VII is actively supported by all automakers, some have expressed concern about the ability of the government to deploy and maintain such an extensive infrastructure. Thus far, this uncertainty has made it difficult for automakers to build a business case around features that utilize communicating infrastructure.

A less ambitious (but more readily achievable) alternative that targets high risk locations (e.g. high-incident intersection locations or curve locations that have a large number of road departures) may be more feasible. Deployment of infrastructure to high risk locations will provide immediate safety benefits to early adopters. It would also be highly desirable that any national infrastructure that is deployed be able to support a nationwide security solution and provide positioning assistance in challenging environments.

\subsection{Summary and Conclusions}

In this chapter we have analyzed the characteristics of various communication-based automotive applications in a systematic manner and classified them into several major generic classes. Such an application characterization and classification effort facilitates the design and implementation of a network protocol stack for these applications. We first proposed a rich set of attributes of the applications, including both application characteristics and networking attributes, to better capture the properties of various applications. We then carefully investigated and analyzed the attributes of $16 \mathrm{~V} 2 \mathrm{X}$ applications. We showed that these applications can be categorized into three major classes: short message broadcast type (for safety applications), on-demand short message unicast type (for convenience applications) and large-volume content download/streaming (for commercial applications). Finally, it should be clear that lists of performance metrics and QoS requirements for each type of application have to be developed; these are used to evaluate the performance trend of applications and network protocols.

The analysis of application characteristics and networking attributes, the classification of various vehicular communication applications, and the need for key performance metrics for each category of applications presented in this chapter, shed some light on our future task of developing a network protocol stack for various communication-based automotive applications. As the next step, the current effort of investigating the potential network solutions for these seven generic types of vehicle-related communication applications should continue, with the consideration of reusability of network protocol modules (or building blocks). To be specific, one should look into designs that decompose the network protocol stack into a set of mechanistic building blocks for different types of applications, so as to maximize the reusability of common building blocks for various applications.

In the development and deployment of communication-based V2X systems, there will be many other challenges to overcome. Some of the challenges that are ahead relate to the evolution of communication technology, positioning, and application specification and development. Deployment of a small number of driver assistance applications early in the next decade would facilitate development of additional applications afterwards that 
could take advantage of increased market penetration and the increasing availability of communication infrastructure.

\section{Acknowledgements}

The authors would like to thank our colleagues, C. Christopher Kellum, Donald K. Grimm, Priyantha Mudalige, Tamer Elbatt, and Varsha Sadekar, for their insightful discussion in the early phase of this work.

\section{References}

ABI Research (2009) Consumer Navigation Systems and Devices, The Changing Dynamics of Portable, Converged, OEM and Aftermarket Navigation. http://abiresearch.com.

Anda, J., LeBrun, J., Ghosal, D., Chuah, C.N. and Zhang, M. (2005) VGrid: Vehicular Ad Hoc Networking and Computing Grid for Intelligent Traffic Control. Proceedings of the $61^{\text {st }}$ IEEE Vehicular Technology Conference (VTC-2005-Spring), Stockholm, Sweden.

Bai, F., Sadagopan. N. and Helmy. A. (2003) The IMPORTANT Framework for Analyzing the Impact of Mobility on Performance of Routing for Ad Hoc Networks. Ad hoc Networks 1(4), 383-403.

Chen, W. and Cai, S. (2005) Ad Hoc Peer-to-Peer Network Architecture for Vehicle Safety Communications. IEEE Communications Magazine 43(4), 100-107.

Chennikara-Varghese, J., Chen, W., Altintas, O. and Cai, S. (2006) Survey of routing protocols for inter-vehicle communications. Proceedings of the $3^{\text {rd }}$ Annual International Conference on Mobile and Ubiquitous Systems - Workshops (mobiquitous).

CICAS (2009) Cooperative Intersection Collision Avoidance Systems (CICAS), USDOT Major Initiative. http://www.its.dot.gov/cicas/index.htm.

Das, S., Nandan, A. and Pau, G. (2004) SPAWN: A Swarming Protocol for Vehicular Ad Hoc Networks. Proceedings of the $1^{\text {st }}$ ACM International Workshop on Vehicular ad hoc Networks (VANET 2004), Philadelphia, PA, USA.

ElBatt, T., Goel, S., Holland, G., Krishnan, H. and Parikh, J. (2006) Cooperative Collision Warning Using Dedicated Short Range Wireless Communications. Proceedings of the $3^{\text {rd }}$ ACM International Workshop on Vehicular ad hoc Networks (VANET 2006), Los Angeles, CA, USA.

FCC (2003) Federal Communications Commission Notice of Proposed Rulemaking and Order FCC 03-324 NPRM.

IEEE 802.11 (1999) IEEE 802.11 WG, Part 11: Wireless LAN Medium Access Control (MAC) and Physical Layer (PHY) Specifications IEEE 802.11 standards.

IEEE P802.11p/D6.0 (2009) Draft Standard for Information Technology - Telecommunications and information exchange between systems - Local and metropolitan area networks - Specific requirements, Part 11: Wireless LAN Medium Access Control (MAC) and Physical Layer (PHY) specifications. Amendment 7: Wireless Access in Vehicular Environments IEEE 802.11 WG.

Korkmaz, G., Ekici, E., Ozguner, F. and Ozguner, U. (2004) Urban multi-hop broadcast protocol for inter-vehicle communication systems. Proceedings of the $1^{\text {st }}$ ACM International Workshop on Vehicular ad hoc Networks (VANET 2004), Philadelphia, PA, USA.

Kosch, T. (2005) Phase-Transition Phenomena with Respect to the Penetration Rate of DSRC Enabled Vehicles. Proceedings of the $12^{\text {th }}$ World Congress on Intelligent Transport Systems (ITS 2005), San Francisco, CA, USA.

LeBrun, J., Chuah, C.N., Ghosal, D. and Zhang, H.M. (2005) Knowledge-based opportunistic forwarding in vehicular wireless ad hoc networks. Proceedings of the $61^{\text {st }}$ IEEE Vehicular Technology Conference (VTC-2005-Spring), Stockholm, Sweden. 
Lin, G., Noubir, G. and Rajaraman, R. (2004) Mobility Models for Ad hoc Network Simulation. Proceedings of the INFOCOM 2004.

Lochert, C., Mauve, M., Füssler, H. and Hartenstein, H. (2005) Geographic routing in city scenarios. Mobile Computing and Communications Review 9(1), 69-72.

Nandan, A., Das, S., Pau, G., Gerla, M. and Sanadidi, M. (2005) Cooperative downloading in Vehicular Ad Hoc Wireless Networks. Proceedings of the $2^{\text {nd }}$ Wireless On-Demand Network Systems and Services (WONS'05), St. Moritz, Switzerland.

Picconi, F., Ravi, N., Gruteser, M. and Iftode, L. (2006) Probabilistic Validation of Aggregated Data for V2V Traffic Information Systems. Proceedings of the $3^{\text {rd }}$ ACM International Workshop on Vehicular ad hoc Networks (VANET 2006), Los Angeles, CA, USA.

Raya, M., Papadimitratos, P. and Hubaux, J.P. (2006) Securing vehicular communications. IEEE Wireless Communications 13, 8-15.

Reumerman, H.J., Roggero, M. and Ruffini, M. (2005) The Application-based Clustering Concept and Requirements for Intervehicle Networks. IEEE Communications Magazine 43(4), 108-113.

Sengupta, R., Rezaei, S., Shladover, S., Cody, D., Dickey, S. and Krishnan, H. (2007) Cooperative Collision Warning Systems: Concept Definition and Experimental Implementation. Journal of Intelligent Transportation Systems 11(3), 143-155.

Shladover, S.E. and Tan, S. (2006) Analysis of Vehicle Positioning Accuracy Requirements for Communication-Based Cooperative Collision Warning. Journal of Intelligent Transportation Systems 10(3), 131-140.

Taliwal, V., Jiang, D., Mangold, H., Chen, C. and Sengupta, R. (2004) Empirical determination of channel characteristics for DSRC vehicle-to-vehicle communication. Proceedings of the $1^{\text {st }}$ ACM International Workshop on Vehicular ad hoc Networks (VANET 2004), Philadelphia, PA, USA.

Torrent-Moreno, M., Jiang, D. and Hartenstein, H. (2004) Broadcast reception rates and effects of priority access in 802.11-based vehicular ad-hoc network. Proceedings of the $1^{\text {st }}$ ACM International Workshop on Vehicular ad hoc Networks (VANET 2004), Philadelphia, PA, USA.

VII (2009) Vehicle Infrastructure Integration (VII), USDOT Major Initiative. http://www.its.dot.gov/vii. VSCC (2005) Vehicle Safety Communications Project, Task 3 Report, Identify Intelligent Vehicle Safety Applications Enabled by DSRC. http://www-nrd.nhtsa.dot.gov/pdf/nrd-12/1665CAMP3web/ images/CAMP3scr.pdf.

VSCC (2006) Vehicle Safety Communications Project Final Report CAMP IVI Light Vehicle Enabling Research Program, DOT HS 810 591. http://www-nrd.nhtsa.dot.gov/pdf/nrd-12/060419-0843/.

$\mathrm{Xu}, \mathrm{Q} ., \mathrm{Mak}, \mathrm{T}$., Ko, J. and Sengupta, R. (2004) Vehicle-to-vehicle safety messaging in DSRC. Proceedings of the $1^{\text {st }}$ ACM International Workshop on Vehicular ad hoc Networks (VANET 2004), Philadelphia, PA, USA.

Yin, J., ElBatt, T.A., Yeung, G., Ryu, B., Habermas, S., Krishnan, H. and Talty, T. (2004) Performance evaluation of safety applications over DSRC vehicular ad hoc networks. Proceedings of the $1^{\text {st }}$ ACM International Workshop on Vehicular ad hoc Networks (VANET 2004), Philadelphia, PA, USA.

Yin, J., Holland, G., Elbatt, T., Bai, F. and Krishnan, H. (2006) DSRC channel fading analysis from empirical measurement. Proceedings of the $1^{\text {st }}$ International Conference on Communications and Networking in China (ChinaCom '06), Beijing, China. 